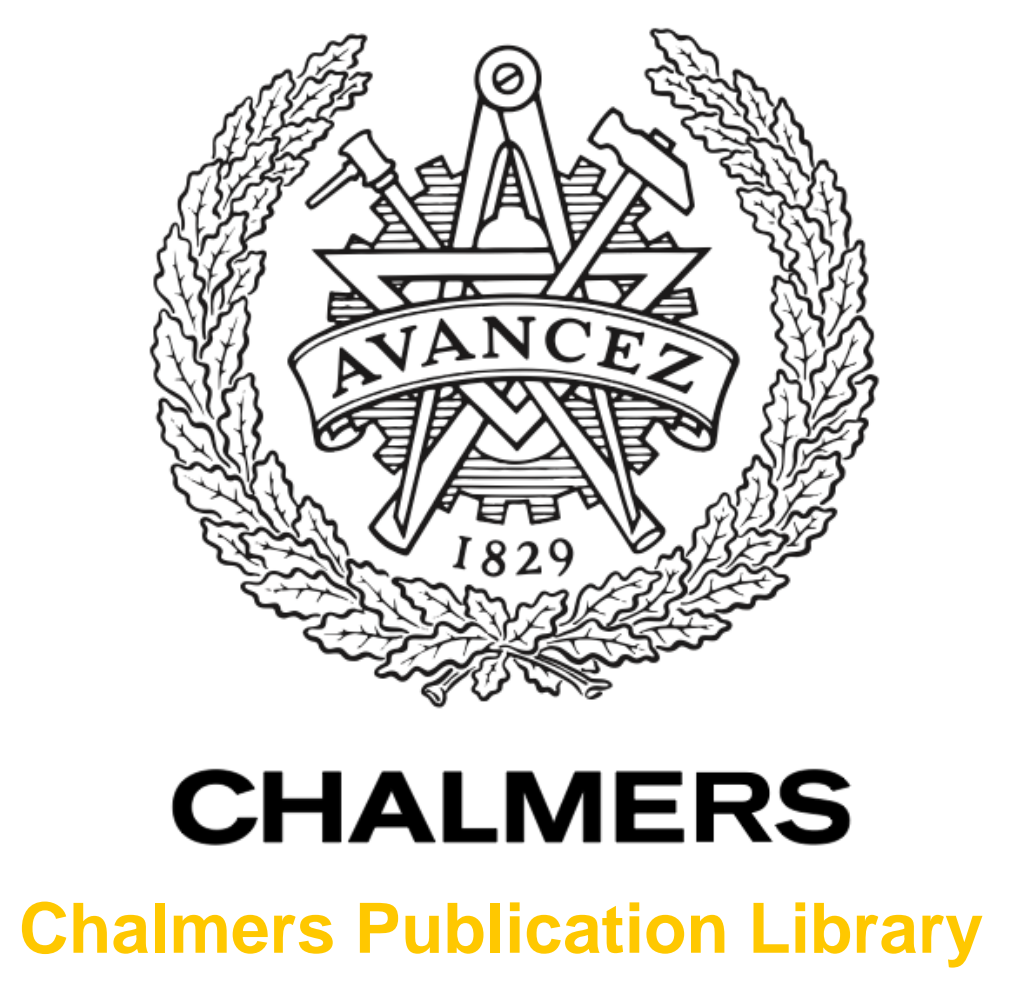

Experimental verification of time-reversal microwave hyperthermia system

This document has been downloaded from Chalmers Publication Library $(\mathrm{CPL})$. It is the author's version of a work that was accepted for publication in:

30th URSI General Assembly and Scientific Symposium, URSIGASS 2011, Istanbul, 13-20 August 2011

Citation for the published paper:

Dobsicek Trefna, H. ; De Berrazueta, J. ; Persson, M. (2011) "Experimental verification of time-reversal microwave hyperthermia system". 30th URSI General Assembly and Scientific Symposium, URSIGASS 2011, Istanbul, 13-20 August 2011

http://dx.doi.org/10.1109/URSIGASS.2011.605135

Down「oaded from: http://publications.lib.chalmers.se/publication/150857

Notice: Changes introduced as a result of publishing processes such as copy-editing and formatting may not be reflected in this document. For a definitive version of this work, please refer to the published source. Please note that access to the published version might require a subscription.

Chalmers Publication Library (CPL) offers the possibility of retrieving research publications produced at Chalmers University of Technology. It covers all types of publications: articles, dissertations, licentiate theses, masters theses, conference papers, reports etc. Since 2006 it is the official tool for Chalmers official publication statistics. To ensure that Chalmers research results are disseminated as widely as possible, an Open Access Policy has been adopted.

The CPL service is administrated and maintained by Chalmers Library. 


\title{
Experimental Verification of Time-Reversal Microwave Hyperthermia System
}

\author{
Hana Dobšíček Trefná, Jaime Peña de Berrazueta, and Mikael Persson \\ Dept. Signal and Systems, Chalmers University of Technology, Hörsalsvägen 11, Gothenburg, Sweden, \\ hanatre@chalmers.se
}

\begin{abstract}
In this contribution, we illustrate the performance of the developed UWB microwave hyperthermia system in combination with the treatment planning tool based on time-reversal algorithm. The experiment comparing the temperature distribution in muscle-equivalent phantom with the predicted SAR pattern was carried out at the ISM radio frequency $434 \mathrm{MHz}$. The obtained results have shown an excellent agreement between planned and measured data in terms of the correct focusing and iso-SAR countours.
\end{abstract}

\section{Introduction}

Hyperthermia is presently used as an adjuvant to the radiation therapy in the treatment of certain types of cancers. Recently, many randomised trials have shown a significant advantage of addition of hyperthermia combined with radiotherapy and/or chemotherapy in the treatment of solid tumours [1]-[3]. The objective of hyperthermia treatment is to raise the temperature in the tumour to a therapeutic level $41^{\circ} \mathrm{C}-45^{\circ} \mathrm{C}$ for a sufficient period of time to achieve cell death or render the cells more sensitive to ionizing radiation and chemical toxins. The present challenge is adequately heating of deep seated tumours while preventing surrounding healthy tissue from undesired overheating and damage.

Presently, the most widely used method of treatment of deep seated tumours is the annular phased array. It is based on an array of radiators placed in a circumferential array around a patient, relying on a constructive wave interference to selectively heat the tumour. The desired wave interference is driven by means of changing the amplitude and phase at the feed-points of the antennas. The right values of these quantities are calculated from a computer simulation where we exploit the time-reversal principle [4].

\section{A TR-Hyperthermia System Prototype}

The prototype has been designed to allow for applications of both continuous and pulsed waves at frequency range of $300 \mathrm{MHz}$ to $1 \mathrm{GHz}$ to enable tumour volume specific treatment. It consist of 12 separate channels with capability to extend it to 24 channels. All of the 12 channels of the system are driven by a common reference excitation signal and the power and the phase of each channel is adjustable, channel-wise, by independent digital phase shifter and voltage controlled power amplifier. In order to ensure accuracy, power level fluctuations and non-linearities associated with signal amplification need to be compensated. A calibration procedure devised in [5] is based on complex S21 parameter measurements using a vector network analyser (VNA).

The present applicator is based on a circular antenna array consisting of 12 elements. We consider two main array configurations: one configuration with 12 antennas placed in the single ring array and one configuration of two antenna rings with 6 antennas in each ring, while one of the rings is turned $30^{\circ}$. The distance between the rings is $55 \mathrm{~mm}$. In order to keep distance in terms of wavelength between radiating elemets and phantom at different frequencies, the diameter of the applicator is adjustable and varies between 110 and $210 \mathrm{~mm}$. 


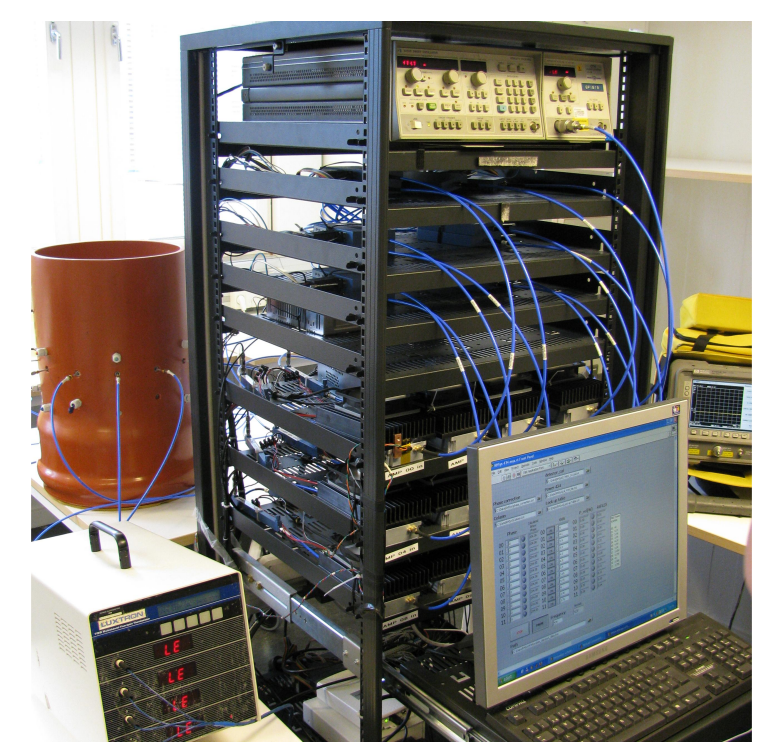

Figure 1: A photo of the proposed hyperthermia system.

\section{Results}

In this experiment, the antenna applicator with 12 elements placed in a single ring array with diameter $22 \mathrm{~cm}$, was used to heat a homogeneous muscle phantom $\left(\epsilon_{r}=64, \sigma=0.41 \mathrm{~S} / \mathrm{m}\right)$. The phantom had a diameter of $10 \mathrm{~cm}$ and a length of $36 \mathrm{~cm}$. Both the antenna array and the phantom were immersed in distilled water $\left(\epsilon_{r}=78, \sigma=0.05 \mathrm{~S} / \mathrm{m}\right)$ with the temperature of $20^{\circ} \mathrm{C}$. The experiment was carried out at the ISM radio frequency $434 \mathrm{MHz}$.

The 2D-TR-FDTD treatment planning procedure was applied to determine the amplitude and phase settings for focusing at thr position $35 \times 50 \mathrm{~mm}$, which is located $15 \mathrm{~mm}$ from the centre of the phantom. The coordinate $50 \times 50 \mathrm{~mm}$ is thus considered as phantom center in Figure 2. The application phase was first modelled using 2-D FDTD as well as in 3-D simulation package CST Microwave Studio including a detailed model of the experimental setup. Finally, the planned amplitude and phase settings were applied experimentally to expose the agar phantom using the developed system. Figure 2(d) shows the obtained temperature distribution in cross-section of the phantom after 9 minutes of exposure. A clear temperature rise of about $9^{\circ} \mathrm{C}$ in the designated location is observed.

The evaluation of the SAR distributions for the 2-D and 3-D simulations and the experiment was carried out by comparing the areas enclosed by $50 \%, 75 \%$ and $90 \%$ iso-SAR contours in cross-section through the phantom at $\mathrm{z}=21 \mathrm{~cm}$. The SAR distribution for experimental case was calculated from temperature distribution captured by IR camera after 9 minutes of heating. An excellent agreement between planned and measured data in terms of the correct focusing and iso-SAR countours was achived, see Figures 2(a)-(c). This can also be seen by comparing of the calculated aPA ratio and RTMi for the foci-spot of radius 10.6 $\mathrm{mm}$ (the $75 \%$ iso-SAR in 2-D) [4]. Although the 2-D modeling overestimates the quality of focusing, the results presented in Table 1 are still surprisingly coherent.

\begin{tabular}{c||ccc}
\hline & 2-D simulation & 3-D simulation & IR camera \\
\hline aPA & 5.40 & 3.31 & 3.28 \\
RTMi & 0.80 & 0.89 & 0.87 \\
\hline
\end{tabular}

Table 1: The aPA ratio and RTMi calculated for different stages of treatmnet planning. 


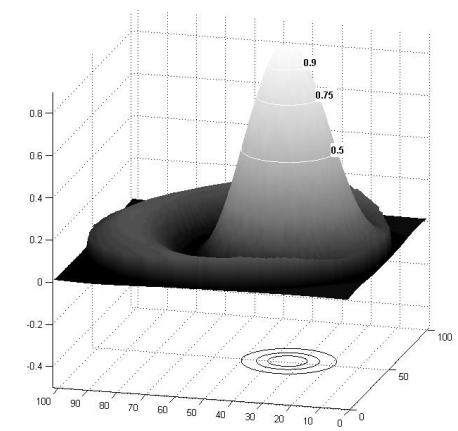

(a)

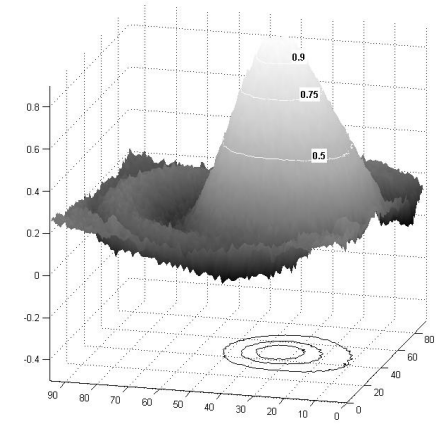

(c)

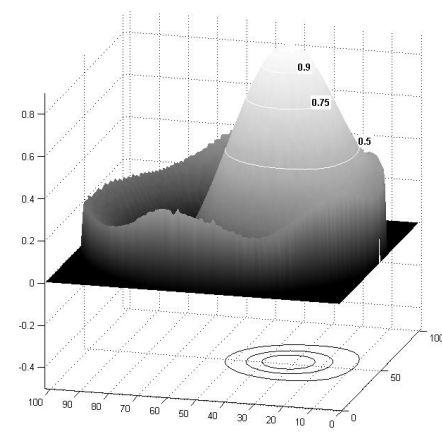

(b)

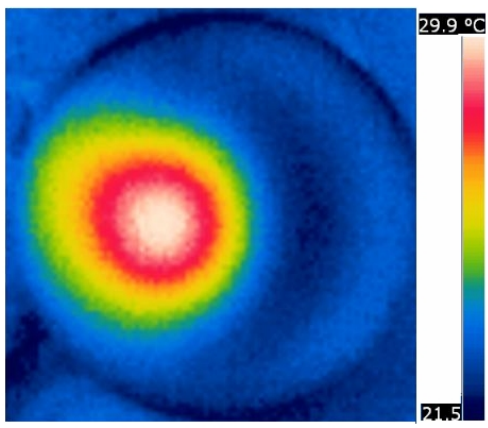

(d)

Figure 2: Normalized SAR distributions obtained from (a) 2-D FDTD planning. (b) full 3-D model in CST. (c) calculated from temperature distribution obtained by IR camera.(d) Temperature distribution obtained by IR camera.

\section{Conclusion}

The obtained results have shown an excellent agreement between planned and measured data in terms of the correct focusing and iso-SAR countours. These promising results encourage the next step; the clinical study.

\section{References}

[1] J. Van der Zee, D. Gonzáles Gonzáles, et al.," Comparison of radiotherapy alone with radiotherapy plus hyperthermia in locally advanced pelvic tumours: a prospective, randomised, multicentre trial", Lancet, vol. 355, pp. 1119-1125, 2000.

[2] E.L. Jones, J.R. Oleson, et al.," Randomized Trial of Hyperthermia and Radiation for Superficial Tumors", J Clin Oncol., vol. 23, pp. 3079-3085, 2005.

[3] R. Colombo, et al.,"Multicentric Study Comparing Intravesical Chemotherapy Alone and With Local Microwave Hyperthermia for Prophylaxis of Recurrence of Superficial Transitional Cell Carcinoma.", J Clin Oncol., vol. 21, pp. 4270-4276, 2003.

[4] H. Dobšíček Trefná, J. Vrba, and M. Persson, "Time-Reversal Focusing in Microwave Hyperthermia for Deep-Seated Tumours", Phys Med Biol, vol 55, pp. 2167-85, 2010.

[5] H. Dobšíček Trefná, P. Togni, R. Shiee, J. Vrba, M. Persson, "Design of a wide-band multi-channel system for time reversal hyperthermia", Submitted to Int J Hyperthermia, 2011. 